\title{
Geographical genetic structure within the human lung fluke, Paragonimus westermani, detected from DNA sequences
}

\author{
D. BLAIR ${ }^{*}$, T. AGATSUMA ${ }^{2}$, T. WATANOBE ${ }^{2}$ M. OKAMOTO ${ }^{3}$ and A. ITO ${ }^{4}$ \\ ${ }^{1}$ Department of Zoology and Tropical Ecology, James Cook University, Townsville, Qld 4811, Australia \\ ${ }^{2}$ Department of Bioresource Chemistry, Obihiro University of Agriculture and Veterinary Medicine, Obihiro, Hokkaido, \\ Fapan \\ ${ }^{3}$ The Institute of Experimental Animal Sciences, Osaka University Medical School, Suita, Osaka 565, Fapan \\ ${ }^{4}$ Department of Parasitology, Gifu University School of Medicine, Gifu 500, Japan
}

(Received 24 February 1997; revised 28 April 1997; accepted 28 April 1997)

\section{SUMMARY}

Nucleotide sequences were obtained for the second internal transcribed spacer of the ribosomal gene repeat and for part of the mitochondrial cytochrome $c$ oxidase subunit I gene from geographical isolates of Paragonimus westermani from Japan, China, Korea, Taiwan, the Philippines, peninsular Malaysia and Thailand. Sequences were obtained from several other species of Paragonimus for comparative purposes. Two groups were recognized within P. westermani: an NE group (China, Japan, Korea, Taiwan) which was relatively uniform and included both diploid and triploid forms, and a southern group (Malaysia, Thailand, Philippines), members of which were genetically distant from one another. According to both ITS2 and COI data, genetic distances among $P$. westermani isolates equalled or exceeded those between some distinct species of Paragonimus. The ITS2 sequences were conserved relative to COI sequences. Substitutions among the latter may be approaching saturation within the genus Paragonimus.

Key words: genetic structure, mitochondrial cytochrome $c$ oxidase subunit I, Paragonimus westermani, phylogenetic trees, nuclear ribosomal second internal transcribed spacer.

\section{INTRODUCTION}

The lung fluke, Paragonimus westermani (Kerbert, 1878), was first described from the lungs of a tiger which died in Amsterdam Zoo in 1877. The geographical origin of the tiger is unclear, although Miyazaki (1978) has stated that it came from India. Human infections with lung fluke are acquired by eating fresh- or brackish-water crustaceans containing live metacercariae or by ingesting raw meat from mammals which act as paratenic hosts. The species has been reported from Pakistan east to southeastern Russia and south to Sumatra, the Philippines and possibly Papua New Guinea. Given this extensive range, and the medical significance of the species, $P$. westerman $i$ has been the focus of much taxonomic and biological study in the past. Early taxonomic studies have been summarized by Yokogawa, Cort \& Yokogawa (1960). Several names in the literature might represent synonyms of $P$. westermani. These include $P$. ringeri (Cobbold, 1880) Ward \& Hirsch, 1915, P. edwardsi Gulati, 1926, $P$. macacae Sandosham, 1954 (see Miyazaki, 1956) and P. asymmetricus Chen, 1977 (see Liu et al. 1982).

* Corresponding author: Department of Zoology and Tropical Ecology, James Cook University, Townsville, Qld 4811, Australia.

Tel: 07781 4322. Fax: 07725 1570. E-mail: David.Blair@jcu.edu.au.
Taxonomic subdivision of $P$. westermani was proposed following the discovery of a triploid, parthenogenetically reproducing strain, often sympatric with sexually reproducing diploids, in Japan, Korea (diploids absent), Taiwan and N.E. China. The triploid form is more pathogenic than the diploid. Miyazaki (1978) proposed the name $P$. pulmonalis for this form.

Subspecies within $P$. westermani have also been proposed. Paragonimus westermani ichunensis Chung, Hsu and Kao, 1978 was erected for worms from N.E. China and the adjacent part of Russia. Paragonimus westermani filipinus was named by Miyazaki \& Habe (1979) for the strain occurring in the Philippines. This strain had previously been given full specific status by 2 groups of workers (i.e. $P$. filipinus Miyazaki, 1978 and $P$. philippinensis Ito, Yokogawa, Araki and Kobayashi, 1978: see Miyazaki, 1981). Diploids from Taiwan were also referred to this subspecies by Miyazaki \& Chiu (1980). Miyazaki (1983) proposed the name $P$. westermani japonicus for diploid forms from Japan. Miyazaki (1991) placed all other diploid forms in the nominate subspecies, $P$. westermani westermani.

Allozyme electrophoresis (reviewed by Blair, 1993) has been used to examine genetic structure within $P$. westermani. Peninsular Malaysian and Philippine isolates are genetically rather distant from one another, but both are very distant from diploid 
Table 1. Material used, geographical origin and gene sequences obtained

(GenBank accession numbers are given.)

\begin{tabular}{|c|c|c|c|}
\hline Species & Origin & ITS2 & $\mathrm{COI}$ \\
\hline \multirow[t]{11}{*}{ P. westermani $(2 \mathrm{n})$} & Hyogo, Japan & U96907 & U97205 \\
\hline & Ohita, Japan & (U96907)* & - \\
\hline & Chiba, Japan & (U96907)* & U97207 \\
\hline & Mie, Japan & $(\mathrm{U} 96907)^{*}$ & U97208 \\
\hline & Minqing, Fujian, China & (U96907)* & U97209 \\
\hline & Taipin, Taiwan & (U96908)† & - \\
\hline & Karapai, Taiwan & U96908 & - \\
\hline & Leyte, Philippines & U96910 & U97213 \\
\hline & Thailand & - & U97212 \\
\hline & Kuala Pilah, Malaysia & - & U97211 \\
\hline & Sungai Wa, Malaysia & U96909 & U97210 \\
\hline \multirow[t]{3}{*}{ P. westermani (3n) } & Tsushima, Japan & $(\mathrm{U} 96907)^{*}$ & (U97205)† \\
\hline & Amakusa, Japan & $(\mathrm{U} 96907)^{*}$ & U97206 \\
\hline & Bogil Island, Korea & $(\mathrm{U} 96907)^{*}$ & $(\mathrm{U} 97205) \dagger$ \\
\hline P. ohirai & Tanegashima, Japan & U96911 & U97214 \\
\hline P. miyazakii & Miyazaki, Japan & U96912 & U97215 \\
\hline P. skrjabini & China, Sichuan & U96913 & U97216 \\
\hline
\end{tabular}

* ITS2 sequence identical to that of $P$. westermani from Hyogo, Japan.

+ ITS2 sequence identical to that of $P$. westermani from Karapai, Taiwan.

$\ddagger$ Partial COI nucleotide sequence identical to that of $P$. westermani from Hyogo, Japan.

strains from Taiwan, Japan and N.E. China. Populations from these three countries are relatively similar to one another and to triploids in the same region.

There are clear geographical differences in host specificity and other biological features. Strains from the Philippines and peninsular Malaysia utilize thiarid snails whereas those in China, Japan, Korea and Taiwan utilize pleurocercid snails (Davis et al. 1994). There are also differences in the extent to which geographical strains utilize various mammals as paratenic, rather than definitive, hosts. $P$. westermani in the Philippines matures readily in rats (Miyazaki \& Habe, 1979), which elsewhere are usually paratenic hosts. Cats and dogs, usually excellent experimental hosts, are relatively poor hosts in Malaysia (Habe, 1987).

In this paper, geographical structuring within $P$. westermani is investigated using data from DNA sequences. These were obtained for the second internal transcribed spacer (ITS2) of the nuclear ribosomal gene repeat and for part of the mitochondrial cytochrome $c$ oxidase subunit I gene (COI).

\section{MATERIALS AND METHODS}

Sources of material used are listed in Table 1. Adult worms were raised in experimental animals. DNA extraction and purification of mtDNA were as described previously (Agatsuma et al. 1994). A single worm was used in each case. Gene regions were amplified using the polymerase chain reaction
(PCR). For the COI region, the primers used were as described by Bowles et al. (1993). For the ITS2, primers used were $\mathrm{BD} 2$ and $3 \mathrm{~S}$ (Bowles, Blair \& McManus, 1995). An additional primer, A28 (5' GGGATCCTGGTTAGTTTCTTTTCCTCCGC 3'), was sometimes used instead of BD2.

All sequences were determined directly from the PCR products. Cycle sequencing reactions were run on an ABI 373A automated sequencer. PCR primers were used as sequencing primers.

For the COI region, published sequence for Fasciola hepatica (GenBank accession number M93388) was used for comparison (Garey \& Wolstenholme, 1989). Codon usage was derived from the same paper, except that the codon ATA was translated to isoleucine rather than methionine (Bowles, Blair \& McManus, 1992) and AAA was translated to asparagine rather than lysine (Ohama et al. 1990).

Trees showing relationships among the species studied were constructed in TREECON (Van De Peer \& De Wachter, 1991). Numbers of transitions and transversions in pairwise comparisons among COI sequences were calculated using a utility in TREECON.

\section{RESULTS}

We were unable to obtain data from both gene regions from some specimens (Table 1). Alignment of sequences was simple and unambiguous for both gene regions. The ITS2 alignment (Appendix A) 
Table 2. Pairwise differences among ITS2 sequences

(Note that all isolates from Japan, China and Korea had sequences identical to that from Hyogo, Japan. Both Taiwanese isolates had identical sequences.)

\begin{tabular}{llllllll}
\hline \hline Species & Origin & 2 & 3 & 4 & 5 & 6 & 7 \\
\hline P. westermani & 1 Hyogo, Japan & 1 & 7 & 6 & 35 & $25^{*} \dagger$ & $25 \dagger$ \\
& 2 Karapai, Taiwan & & 8 & 7 & 36 & $26 * \dagger$ & $26 \dagger$ \\
& 3 Leyte, Philippines & & & 1 & 38 & $29 * \dagger$ & $29 \dagger$ \\
& 4 Sungai Wa, Malaysia & & & & 37 & $28 * \dagger$ & $28 \dagger$ \\
P. ohirai & 5 & & & & & $27 * \dagger$ & $27 \dagger$ \\
P. miyazakii & 6 & & & & & & $0 *$ \\
P. skrjabini & 7 & & & & & \\
\hline \hline
\end{tabular}

* Ignoring 1 ' $\mathrm{N}$ ' in P. miyazakii.

$\uparrow$ Ignoring a deletion $2 \mathrm{nt}$ long in P. miyazakii and P. skrjabini.
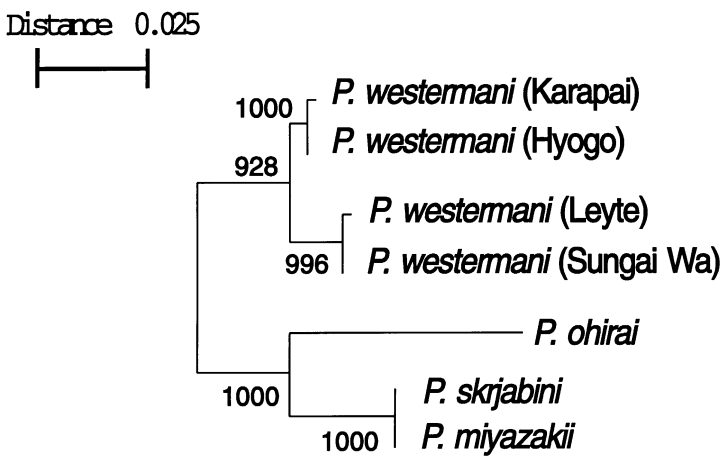

Fig. 1. Tree depicting relationships among Paragonimus species inferred from ITS2 data. A distance matrix was calculated using the Kimura 2-parameter model and the tree constructed using the neighbour-joining approach in TREECON. The data set was bootstrapped 1000 times and the appropriate bootstrap values placed on each branch.

was 363 bases in length. Identical ITS2 sequences were obtained from all Japanese, Korean and Chinese $P$. westermani isolates, whether diploid or triploid. Only the diploid from Hyogo is included in Table 2 as a representative of these isolates. Specimens from Taipin and Karapai (both Taiwan), were identical to each other but differed from Japanese specimens at 1 site. Malaysian and Philippine sequences differed from one another at a single site but had 6 sites in common to the exclusion of samples from other countries (Table 2). Figure 1 shows relationships among the isolates used as inferred from their ITS2 sequences.

The COI alignment (Appendix B) is 393 bases long including an insertion of one codon $(3 \mathrm{nt})$ in the outgroup, $F$. hepatica, relative to all the Paragonimus species. This insertion was excluded from all calculations. In contrast to the ITS2 data, only 3 isolates of $P$. westermani exhibited identical COI nucleotide sequences. These were the diploid from Hyogo (Japan) and triploids from Tsushima (Japan) and Bogil Island (Korea). Other isolates from Japan differed from these by only a single transitional change and the sample from Minqing, China differed at 6 sites. Sequences from peninsular Malaysia, the Philippines and Thailand differed from Japanese sequences, and from each other, at many more sites, with most of the changes being transitions (Table 3). All $P$. westermani isolates differed at many sites from other members of the genus and from $F$. hepatica (Table 3) and transition:transversion ratios were much lower than among $P$. westermani isolates. The tree shown in Fig. 2 shows relationships among isolates based on COI DNA sequences.

The many nucleotide substitutions seen among members of the genus Paragonimus are almost all synonymous. The maximum number of amino acid differences among Paragonimus species is 4, as opposed to 20-22 differences between $F$. hepatica and Paragonimus species.

\section{DISCUSSION}

Paragonimus westermani is partitioned into at least 2 groups according to both ITS2 and COI sequences. One group, the N.E. group, consists of diploids and triploids from China, Japan, Taiwan and Korea and exhibits relatively little molecular variation. The second group, the southern group, is less clearly defined, especially from the COI data. It consists of isolates from Malaysia, Thailand and the Philippines. Based on the COI data, each isolate in this group is almost as distant from the others as from any member of the N.E. group. The two groups are also distinguished by a major biological feature - the family of snail hosts utilized. Members of the southern group utilize thiarids as far as is known, and members of the N.E. group utilize pleurocercids (Davis et al. 1994).

The genetic divisions identified from our data correspond only in part to taxonomic subdivisions proposed previously. The N.E. group, comprising diploids and triploids from Japan, Taiwan, Korea and China, does not show strong substructuring. The triploid form (P. pulmonalis of Miyazaki, 1978) is not distinct from sympatric diploids. Recognition of the name $P$. pulmonalis would therefore render $P$. 


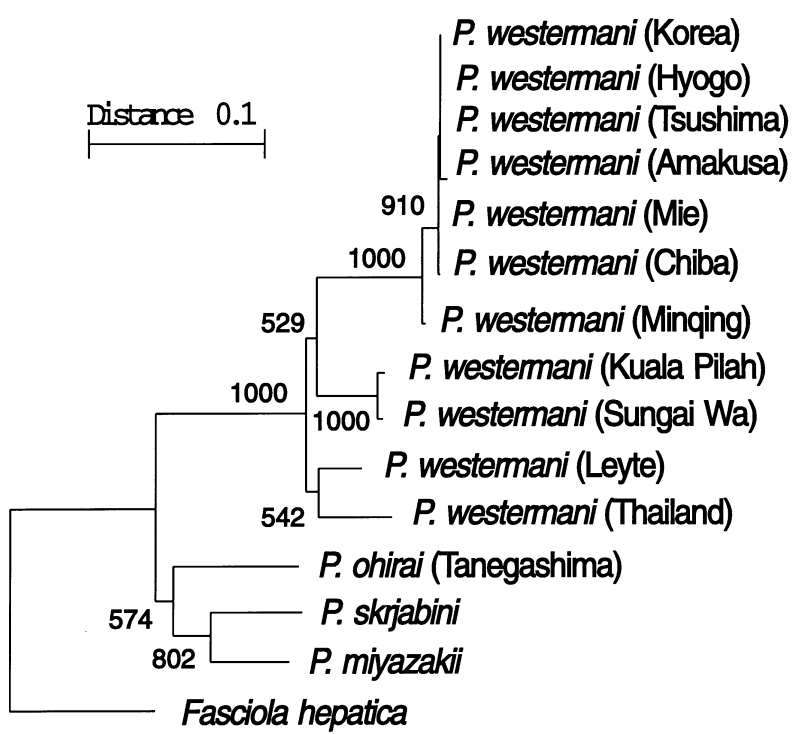

Fig. 2. Tree depicting relationships among Paragonimus species inferred from COI nucleotide data. A distance matrix was calculated using the Kimura 2-parameter model and the tree constructed using the neighbourjoining approach in TREECON. The data set was bootstrapped 1000 times and the appropriate bootstrap values placed on each branch. Fasciola hepatica was used as an outgroup taxon.

westermani paraphyletic. The Taiwanese diploids we sampled are allied with the N.E. group according to the sequence data. Affinities with populations from the Philippines, suggested by Miyazaki \& Chiu (1980), are not supported by our sequence data.

According to Miyazaki (1991), P. westermani populations from the Philippines belong to one subspecies ( $P$.w. filipinus) and those from Malaysia and Thailand to another (P.w. westermani). Genetic differences (based on the COI data) between isolates from these locations are considerable. However, the Thai isolate is slightly closer to the Philippine isolate than to the Malaysian. These COI differences are almost as great as between two distinct species, $P$. skrjabini and P. miyazakii. Differences between ITS2 sequences were rather few. The fact that any intraspecific ITS2 differences were noted is surprising. Intraspecific variation in ITS2 sequences among trematodes is virtually unknown. For example, Morgan \& Blair (1995) found no differences in ITS2 sequences between samples of Echinostoma revolutum from Germany and Indonesia. Similarly, Després et al. (1992) found no differences in ITS2 sequences among samples of Schistosoma mansoni from many sites in Africa and the Americas. Furthermore, several species within the Schistosoma haematobium group had identical sequences. In this study, we found identical ITS2 sequences shared by P. skrjabini and P. miyazakii. Genetic differentiation among $P$. westermani populations may be sufficient to merit eventual recognition of distinct species (and see Agatsuma et al. 1993). However, more data are required to support this notion, and especially data 
from nominal $P$. westermani populations in Indonesia, Sri Lanka, India and as-yet unsurveyed regions of China.

Agatsuma and co-workers (reviewed by Blair, 1993), noted that all populations of the parthenogenetic triploid form of $P$. westermani were electrophoretically identical. This implied a single origin of the triploid, perhaps due to hybridization between a Japanese form and one from elsewhere. Agatsuma et al. (1994) suggested that the maternal ancestor of the triploid form might have come from outside Japan because none of the Japanese diploid strains examined had the same mitochondrial DNA restriction fragment pattern as the triploid. Our COI sequence data do not support this view. Given the geographical variation noted among the COI sequences of $P$. westermani, the fact that at least 1 Japanese strain had a COI nucleotide sequence identical with 2 of the triploids suggests a Japanese maternal ancestor. The triploid strain from Amakusa differed at 2 nucleotide sites, one of them non-synonymous, from the remaining triploids. This could be a result of mutations in the Amakusa lineage, or could result from independent origins of the triploid populations from varied ancestors. The data are too few to make speculation worthwhile.

Of the 2 gene regions used, the ITS2 is clearly the more conserved. Sequences from P. skrjabini from China and P. miyazakii from Japan were identical. Differences among $P$. westermani isolates were few, and among Paragonimus species, substitutions occurred generally at fewer than $10 \%$ of sites. Saturation is not likely to have occurred in this region. The ITS2 is likely to be a good marker for specific relationships within the genus Paragonimus, but less useful for investigating intra-specific genetic structure.

The COI gene exhibits many more variable sites and gives a clear indication of genetic structure within $P$. westermani. It also distinguishes between P. skrjabini and P. miyazakii whereas the ITS2 does not. Among the more divergent isolates of $P$. westermani, over $10 \%$ of sites exhibit substitutions and the transition:transversion (TS:TV) ratio is about 10:1. Among species of Paragonimus, substitutions occur at up to $20 \%$ of sites and TS:TV ratios can be lower than 2:1. Absolute numbers of transitions are similar in comparisons among Paragonimus species and $F$. hepatica (although in comparisons with $F$. hepatica, the TS: TV ratio is about $1: 1)$. This suggests that saturation is being approached, at least for transitions, within the genus Paragonimus. Since almost all substitutions among Paragonimus sequences are synonymous, only the third (and a few first) codon positions are free to change and saturation is probably reached quickly. The phylogenetic resolving power of the COI gene might be considerably reduced when distant species in the same genus are included.

We wish to thank Dr Hiromu Sugiyama, Department of Parasitology, National Institute of Health, Japan, Dr Shigehisa Habe, Department of Parasitology, Fukuoka University and Dr Shibaha, Laboratory Animal Research Center, Tottori University for specimens of $P$. ohirai and $P$. westermani. Dr Kenjiro Kawashima, Kyushyu University, provided encouragement throughout our study. Funding was provided by the Australian Research Council (D. B.) and by the Japanese Government (T. A.) (08670272).

\section{A P PENDIX A. Alignment of ITS2 sequences.}

(Alignment gaps indicated by a hyphen. Sites with a nucleotide identical to that on the top line indicated with a dot.)

PwH
PwK
PwSW
PwL
Po
Pmi
Psk
//
PwH
PwK
PwSW
PwL
Po
Pmi
Psk
//
PwH
PwK
PwSW
PwL
Po
Pmi
Psk
$/ /$
PwH
PwK
PwSW
PwL
Po
Pmi
Psk

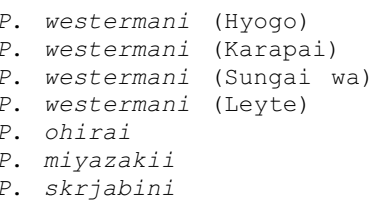


A P PENDIX B. Alignment of partial COI sequences displayed as codons.

(Sites with a nucleotide identical to that on the top line are indicated with a dot.)

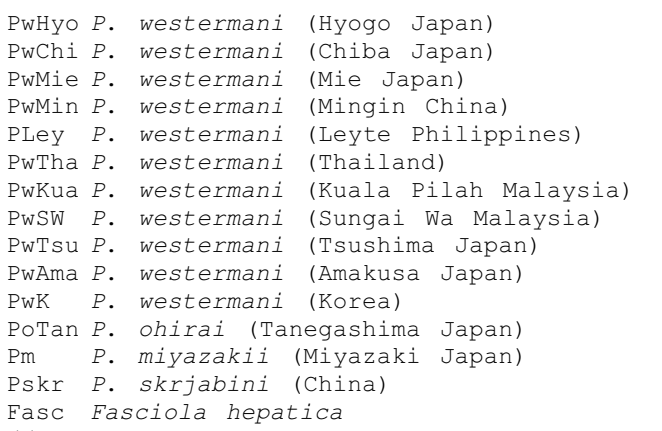

\begin{tabular}{|c|c|c|c|c|c|c|c|c|c|c|c|c|c|c|}
\hline $\mathrm{CTG}$ & $\mathrm{TT}$ & TG & $\mathrm{CT}$ & GG & TTT & GGT & ATC & GTG & AGC & CAC & ATT & TGC & $\mathrm{AlG}$ & $\mathrm{ACC}$ \\
\hline & - & $\cdots$ & $\cdots$ & $\cdots$ & $\cdots$ & . & . & $\ldots$ & $\ldots T$ & $\cdots$ & $\cdots$ & & $\cdots$ & \\
\hline & . & . & . & . & $\cdots$ & . & & $\cdots$ & & $\cdots$ & 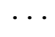 & & & \\
\hline & $\cdot$ & . & . & . & . & . & & . & & $\cdots$ & . & & & \\
\hline & . & $\cdots$ & & . & $\ldots$ & . C & & $\cdots$ & & - & $\ldots C$ & & & \\
\hline & . & . & $\ldots G$ & $\cdots$ & . & $\cdots$ & $\cdots$ & $\cdots$ & $\cdot \mathrm{T}$ & $\cdot$ & $\ldots 0$ & & & \\
\hline & . & . . & $\ldots \mathrm{A}$ & . & $\cdots$ & $\cdots$ & . & $\ldots$ &. $\mathrm{T}$ & $\cdots$ & $\ldots C$ & & $\cdots$ & \\
\hline & . & $\cdots$ & $\ldots A$ & . & $\ldots$ & . & $\cdots$ & $\ldots$ & $\ldots \mathrm{T}$ & $\ldots$ & $\ldots \mathrm{C}$ & & & \\
\hline & & & & & & & & $\cdots$ & • & . & - & & & \\
\hline & 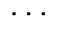 & & & & . & & & . &. $\mathrm{T}$ & . & $\ldots \mathrm{C}$ & & & \\
\hline & & & . A & & . & & & . & & $\cdots$ & . C & & & \\
\hline & & $\ldots A$ & $\ldots A$ & A & . & $\ldots G$ & . & $\cdot$ & $\ldots \mathrm{A}$ & $\ldots T$ & . & . . & & \\
\hline & . & $\ldots$ & $\ldots A$ & $\ldots$ & $\ldots$ & $\ldots G$ & & . & $\ldots$ & $\ldots T$ & & $\ldots 7$ & & \\
\hline & 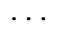 & $\ldots$ & $\ldots \mathrm{C}$ & . & $\ldots \mathrm{C}$ & $\ldots G$ & & & . 7 & $\ldots T$ & . & $\ldots 7$ & & \\
\hline$\cdots$ & & $\ldots \mathrm{A}$ & $\ldots \mathrm{C}$ & & $\cdots$ & $\ldots G$ & G.T & A.T &. $\mathrm{T}$ &. $\mathrm{T}$ & $\cdots$ & $\ldots T$ & $\cdots$ & \\
\hline
\end{tabular}
Fasc

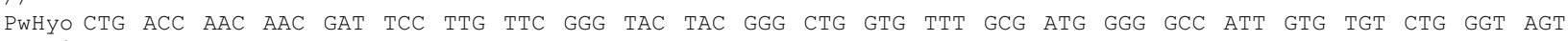

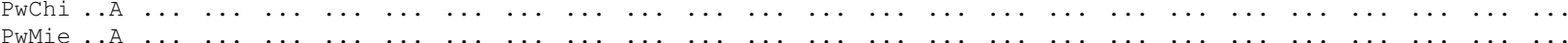

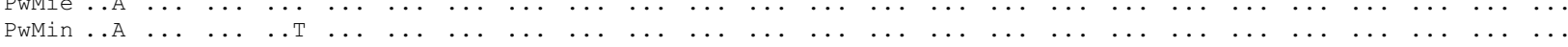

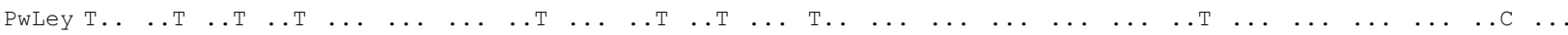

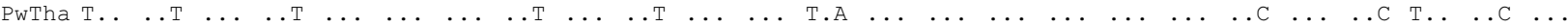

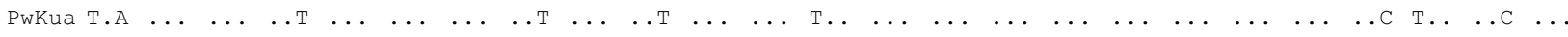

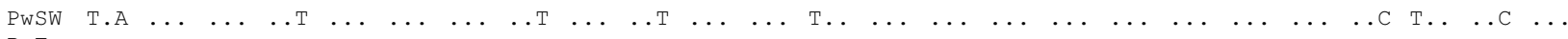

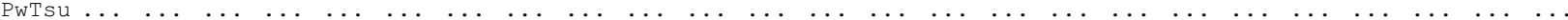

\section{CAMBridge JDURNALS}




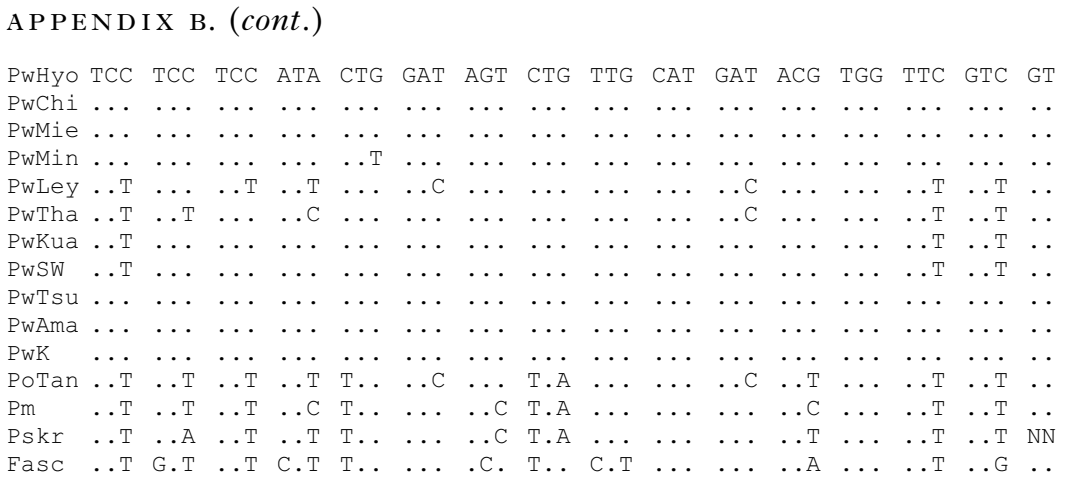

\section{REFERENCES}

Agatsuma, T., LAI, K., OW-YANG, C. K., HABE, S., SUgiYAMA, H., hiRAi, H. \& KAWASHiMA, K. (1993). Genetic differentiation between Malaysian and other Asian Paragonimus westermani. Tropical Biomedicine 10, 45-52.

AGATSUMA, T., YANG, L., KIM, D. \& YONEKAWA, H. (1994). Mitochondrial DNA differentiation of Japanese diploid and triploid Paragonimus westermani. Fournal of Helminthology 68, 7-11.

BLAIR, D. (1993). Molecular variation in fasciolids and Paragonimus. Acta Tropica 53, 277-289.

BOWLES, J., HOPE, M., TIU, W. U. \& LIU, X. S. \& McMANUS, D. P. (1993). Nuclear and mitochondrial genetic markers highly conserved between Chinese and Philippine Schistosoma japonicum. Acta Tropica 55, 217-229.

BOWles, J., BlaiR, D. \& McManus, D. P. (1992). Genetic variants within the genus Echinococcus identified by mitochondrial DNA sequencing. Molecular and Biochemical Parasitolology 54, 165-174.

BOWles, J., Blair, D. \& McManus, D. P. (1995). A molecular phylogeny of the human schistosomes. Molecular Phylogenetics and Evolution 4, 103-109.

DAVIS, G. M., CHEN, C. E., KANG, Z. B. \& LIU, Y. Y. (1994).

Snail hosts of Paragonimus in Asia and the Americas. Biomedical and Environmental Sciences 7, 369-382.

DESPRÉs, L., IMBERT-ESTABLET, D., COMBES, C. \& BONHOMme, F. (1992). Molecular evidence linking hominid evolution to recent radiation of schistosomes (Platyhelminthes: Trematoda). Molecular Phylogenetics and Evolution 1, 295-304.

GAREY, J. R. \& WOLSTENHOLME, D. R. (1989). Platyhelminth mitochondrial DNA: Evidence for early evolutionary origin of a tRNA ser AGN that contains a dihydrouridine-arm replacement loop, and of serine-specifying AGA and AGG codons. Fournal of Molecular Evolution 28, 374-387.

HABE, s. (1987). Experimental infection of mammals with Malaysian P. westermani. In Paragonimus in Asia: Biology, Genetic Variation and Speciation (Paragonimus Research Report No. 1) (ed.
Kawashima, K.), pp. 29-48. Kyushu University of Health Sciences, Fukuoka.

LIU, S., ZHANG, D., LI, Y. \& XU, Z. (1982). Observations on the variation of the metacercaria of Paragonimus westermani. Kexue Tongbao 27, 560-562.

miYazaki, I. (1956). Re-examination of Paragonimus macacae Sandosham, 1953, most probably a synonym of $P$. westermani (Trematoda: Troglotrematidae). Medicine and Biology 40, 35-38 (In Japanese.)

miYazaki, I. (1978). Two types of lung fluke which has been called Paragonimus westermani (Kerbert, 1878). Medical Bulletin of Fukuoka University 5, 251-263.

MiYAZAKI, I. (1981). Taxonomical studies of the lung fluke occurring in the Philippines. Medical Bulletin of Fukuoka University 8, 153-158.

miYaZaki, I. (1983). Paragonimus westermani japonicus $\mathrm{n}$. subsp. occurring in Japan. Medical Bulletin of Fukuoka University 10, 67-76.

miyazaki, I. (1991). Helminthic Zoonoses. Tokyo, International Medical Foundation of Japan.

MIYAZAKI, I. \& CHIU, J. K. (1980). Examination of the socalled 'Paragonimus westermani' in Taiwan. Medical Bulletin of Fukuoka University 7, 277-279.

MiYazaki, I. \& Habe, s. (1979). Paragonimus westermani filipinus Miyazaki, 1978, stat. n. occurring at Jaro, Leyte, The Philippines. Medical Bulletin of Fukuoka University 6, 447-462.

MORGAN, J. A. \& BLAIR, D. (1995). Nuclear rDNA ITS sequence variation in the trematode genus Echinostoma: an aid to establishing relationships within the 37-collar-spine group. Parasitology 111, 609-615.

OHAMa, T., OWASa, S., Watanabe, K. \& JUKES, T. H. (1990). Evolution of the mitochondrial genetic code IV. AAA as an asparagine codon in some animal mitochondria. Fournal of Molecular Evolution 30, 329-332.

VAN DE PEER, Y. \& DE WACHTER, R. (1993). TREECON: a software package for the construction and drawing of evolutionary trees. Computer Applications in the BioSciences 9, 177-182.

yokogawa, s., CORT, W. \& YoKogaWa, M. (1960). Paragonimus and paragonimiasis. Experimental Parasitology 10, 81-137, 139-205. 